\title{
Modelagem geoestatística aplicada a geologia de engenharia
} Geostatistic modelling applied to engineering geology

\author{
BRUNO RODRIGUES OLIVEIRA ${ }^{1}$, GABRIELA BRANQUINHO ANTÔNIO ${ }^{1}$
}

1Universidade de Brasília, UnB - brunorodriguesoli@gmail.com,gabrielabranquinho56@gmail.com

\begin{abstract}
Resumo
A atuação da geologia na área da engenharia civil vem crescendo muito nos últimos anos devido ao aquecimento do setor, entretanto os estudos geológicos-geotécnicos demandam muito tempo e são dispendiosos, todavia é possível utilizar a geoestatística para gerar informações que otimizem a elaboração desses estudos. Este artigo tem como objetivo utilizar a geoestatística para produção de informações com a finalidade de auxiliar no planejamento e/ou complementar os estudos geológicos aplicados a engenharia. Primeiramente, realizou-se uma análise exploratória dos dados, a espessura do solo superficial na área de estudo, que servirão como base para o estudo. Em seguida foi realizada a modelagem geoestatística objetivando compreender o condicionamento espacial e a variabilidade dos dados e construção do grid de estimativa. Por fim, foram estimadas espessuras de solo superficial no grid pelo método de krigagem ordinária. Essa metodologia, permitiu, a partir dos dados coletados, elaborar mapas temáticos visando auxiliar a estudos geológico-geotécnicos que venham a ser realizados na área de estudo e apresentar uma forma de aplicação da geoestatística na geologia de engenharia.
\end{abstract}

Palavras-chave: Geoestatística; Krigagem; Geologia de Engenharia

\begin{abstract}
The actuation of the geology in civil engineering field has been growing significantly in recent years due to the heating sector, however the geological and geotechnical studies demand long time and are expensive, however you can use geostatistics to produce information that optimize the preparation of these studies. This article intends to use the geostatistics to produce information in order to assist in planning and / or complementing the geological studies applied to engineering. First, an exploratory analysis of data was performed, the thickness of the Superficial soil in the study area, which will serve as basis for the study. Then, it was performed a geostatistical modeling with the objective of understanding the conditioning space and the variability of the data and the construction of the estimation grid. At last, the soil thicknesses was estimated on the grid using the ordinary kriging method. This methodology allowed, from the collected data, to produce thematic maps to complement the geological and geotechnical studies conducted in the study area and to present a form of application of geostatistics in engineering geology.
\end{abstract}

Keywords: Geostatistics; Kriging; Engineering Geology 


\section{Introdução}

O presente artigo tem como tema apresentar uma aplicação da geoestatística no setor de geologia de engenharia como uma ferramenta capaz de otimizar o planejamento e/ou complementar estudos geológico-geotécnicos, principalmente, em meios urbanos.

Nos últimos anos a demanda por profissionais na área de geologia de engenharia vem crescendo e sua atuação é quase sempre direcionada para obras civis de pequeno a médio porte, como redes de abastecimento de água e esgoto.

A aplicação da geologia na área da engenharia civil está associada aos estudos geológicos-geotécnicos que constituem uma análise do meio físico visando identificar as características do meio mais relevantes para a construção de obras civis.

A execução de campanhas de sondagens em áreas urbanizadas enfrentam dificuldades como a falta de locais adequados para realização das investigações, devido a ocupações densas, ou pela existência de redes de utilidades, o que acarreta em uma distribuição irregular das sondagens ao longo da área do empreendimento.

A execução dos estudos supracitados, em sua maioria, demanda muito tempo e são dispendiosos. Nesses casos, a aplicação de métodos geoestatísticos para predição de resultados em locais não amostrados surge como uma alternativa viável e perfeitamente exequível. A geoestatística não é somente a aplicação de métodos estatísticos em geologia, mas o estudo de variáveis regionalizadas, ou seja, variáveis que apresentam um condicionamento espacial (LANDIN, 2006).

A modelagem geoestatística é aplicada neste estudo objetivando analisar e mapear a variável espessura da camada de solo superficial, com uma abordagem probabilística, que permitirá um melhor entendimento do comportamento espacial da mesma, contribuindo assim para tomadas de decisões como na definição de traçados em projetos de abastecimento e/ou saneamento visando aumentar escavações em materiais de primeira categoria, reduzindo assim os custos de implantação de projetos e otimizando o planejamento dos estudos a serem realizados posteriormente.

A metodologia utilizada para a obtenção dos resultados do presente trabalho, de maneira sucinta, será apresentada a seguir e detalhada nos seus respectivos tópicos. Primeiramente, será realizada a apresentação dos dados e em seguida a análise exploratória e modelagem geoestatística dos mesmos para compreender seu condicionamento espacial e sua variabilidade.

Com base na modelagem, será realizada a predição geoestatística pelo método da krigagem ordinária e serão apresentados os resultados e discussões. Por fim, serão apresentadas as conclusões sobre a aplicabilidade da ferramenta e os resultados obtidos.

\section{Contextualização dos dados}

A área urbanizada, próxima ao rio Mundaú, na sede do município de União dos Palmares (Figura 1) foi escolhida como estudo de caso do presente trabalho. O município está localizado na região norte-nordeste do estado de Alagoas, limitando-se a norte com os municípios de São José da Laje e Ibateguara, a sul com Branquinha, a leste com Joaquim Gomes e a oeste com Santana do Mundaú e o acesso a partir da capital do estado, Maceió, é feito através da rodovia pavimentada BR104, com percurso em torno de $77 \mathrm{~km}$.

A área apresenta uma amplitude altimétrica de $20 \mathrm{~m}$ com um relevo plano a suavemente ondulado e está inserida em três unidades pedológicas compostas por Gleissolos, Argissolos Vermelho e/ou Amarelos, entretanto só foram investigados locais na unidade dos Gleissolos. Geologicamente, está totalmente inserida na unidade geológica Belém do São Francisco caracterizada por rochas metamórficas intensamente dobradas e medianamente a intensamente fraturadas (OLIVEIRA \& BRANQUINHO, 2015). 


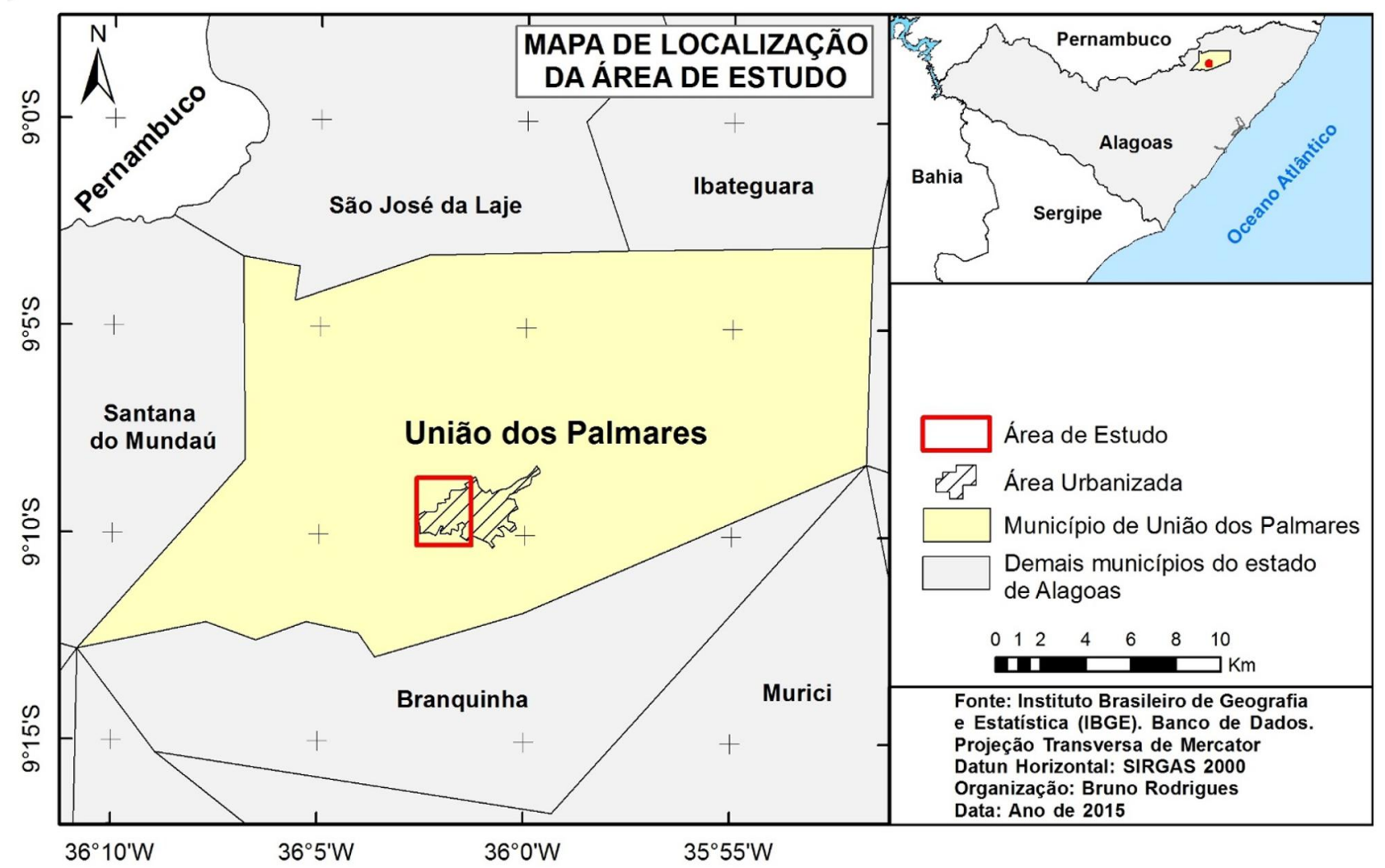

Figura 1 - Mapa de localização da área de estudo (Fonte: Adaptado de OLIVEIRA \& BRANQUINHO, 2015).

As amostras utilizadas para realizar a modelagem geoestatística da espessura de solo superficial da área de estudo foram os perfis de sondagem de 48 investigações executadas durante a elaboração do projeto executivo de saneamento integrado para a prefeitura do município de União dos Palmares, no ano 2015. Como o foco principal do projeto era a determinação da profundidade ao longo dos traçados do sistema de esgotamento sanitário, as sondagens foram realizadas ao longo das vias de acesso da cidade o que acarretou em uma distribuição irregular ao longo da área de estudo como pode ser observado na Figura 2.

Cabe ressaltar que parte das sondagens foram limitadas até $1,50 \mathrm{~m}$ de profundidade, sendo assim, esses perfis de sondagem utilizados não representam a espessura total do solo na área de estudo, mas apenas a camada superficial que é considerada de extrema importância em alguns projetos de engenharia.

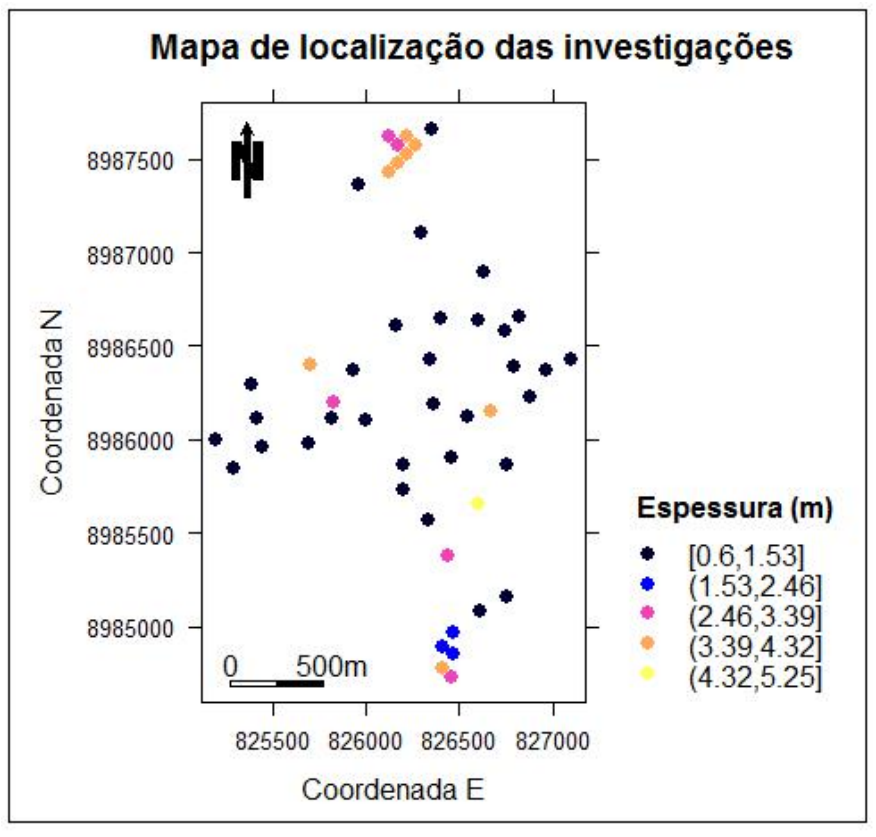

Figura 2 - Mapa de distribuição espacial das amostras na área de estudo. 


\section{Análise exploratória e modelagem geostatística}

Para realizar a análise exploratória dos dados e a modelagem geoestatistica foi utilizado o software $\mathrm{R}$ com os seguintes pacotes: gstat (Spatial and Spatio-Temporal Geostatistical Modelling, Prediction and Simulation); lattice (Trellis Graphics for $R$ ); $s p$ (Classes and Methods for Spatial Data); moments (Moments, cumulants, skewness, kurtosis and related tests); rgdal (Bindings for the Geospatial Data Abstraction Library); e splancs (Spatial and Space-Time Point Pattern Analysis).

A realização da estatística descritiva da variável espessura revelou que as investigações atingiram uma profundidade média de 1,91m com um desvio padrão de $1,05 \mathrm{~m}$. O teste de normalidade de AndersonDarling $\left(A^{2}=7.47\right.$ e Valor de $\left.P<0,0005\right)$ mostra que os dados não apresentam uma distribuição normal, o que é confirmado pelos valores de assimetria e curtose calculados.

Quando o valor de assimetria é maior que zero significa que a distribuição tem uma cauda direita mais pesada, ou seja, que apresentam uma maior quantidade de valores acima da média enquanto o valor de curtose maior que zero significa que a distribuição em questão é mais afunilada e concentrada que a distribuição normal e que obter valores que não se aproximam da média a vários múltiplos do desvio padrão é extremamente fácil.

Os outliers identificados no boxplot são os valores responsáveis pela assimetria observada no histograma e foram mantidos no presente estudo por indicar locais onde a camada de solo superficial se apresenta de fato mais espessa. Todas as informações supracitadas estão resumidas na Figura 3.

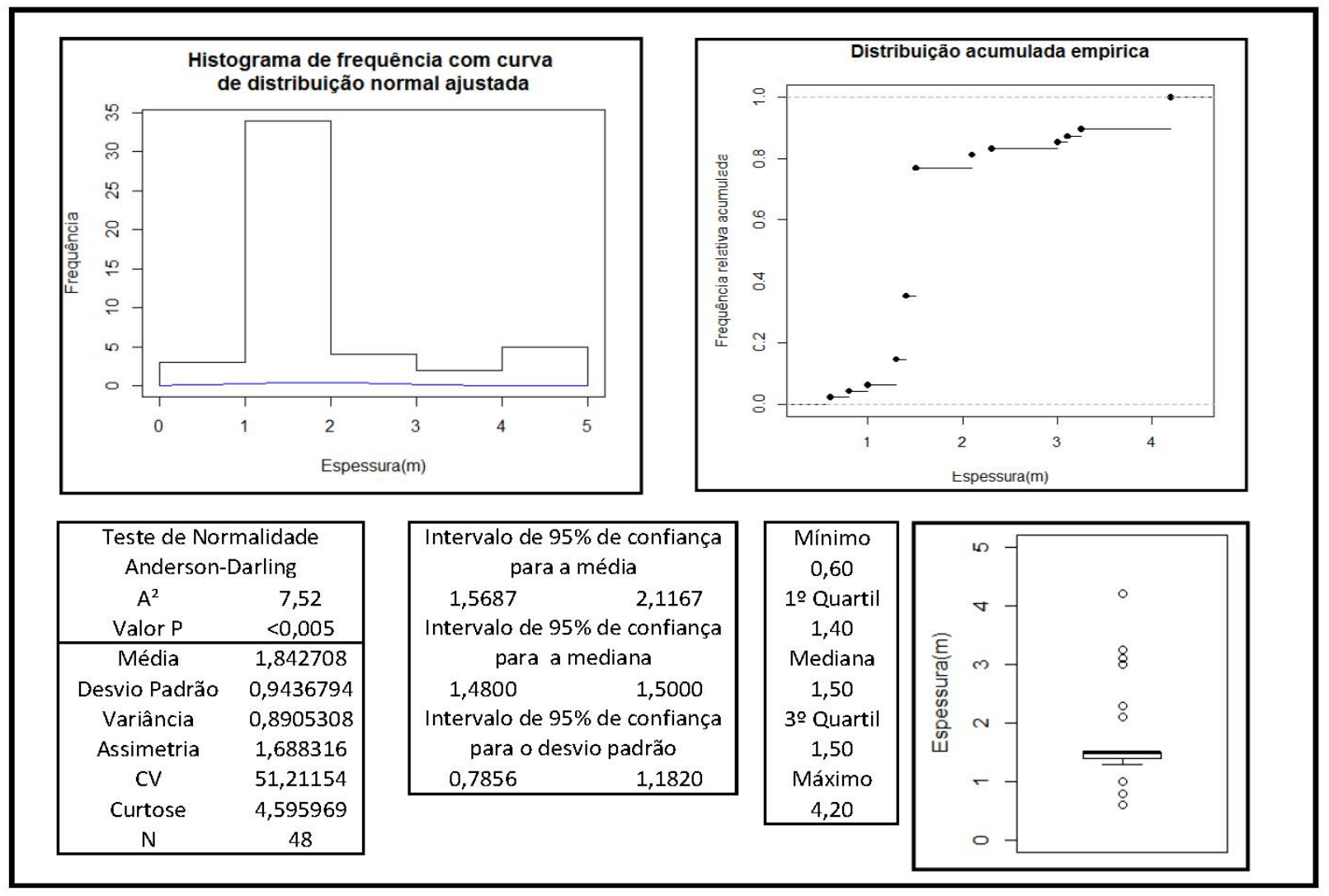

Figura 3 - Resumo da análise estatística univariada realizada para a variável espessura.

Para predizer o valor de um ponto não amostrado é necessário determinar o modelo de correlação espacial da variável regionalizada através de uma análise variográfica. Posteriormente, ajusta-se um modelo teórico que permite determinar o valor de correlação espacial para qualquer distância no espaço amostrado. 
A análise variográfica consiste no cálculo de um semivariograma, que é uma função que mostra a dissimilaridade entre pares de pontos a uma determinada distância (YAMAMOTO \& LANDIM, 2013; BRAGA, 2014) e que aumenta até atingir o patamar, o ponto com maior valor de variância dos dados. Tal análise tem o objetivo de quantificar a variação no espaço do fenômeno regionalizado em estudo e cujos resultados são utilizados para a organização do sistema de equações da krigagem (YAMAMOTO, 2001).

Outros parâmetros interpretados são o alcance, que determina a distância máxima de dependência entre amostras, e o efeito pepita que reflete a variância aleatória comumente atribuído a erros de medição ou a forma de amostragem dos dados.

Para identificar a existência de anisotropia foi elaborado um mapa de semivariância em todas as direções, conhecido como mapa variograma, que permite identificar com facilidade o eixo de variação máxima. Como apresentado na Figura 4, a direção principal de variação dos dados, expressa em ângulos azimutais, é a $175^{\circ}$ e a direção do eixo menor $85^{\circ}$. Todavia, observa-se que a variância dos dados é muito semelhante em todas as direções.

Para calcular a modelagem da variável regionalizada foram calculados variogramas experimentais em diversas direções e um onidirecional para definição do raio para o modelo isotrópico. Os variogramas foram calculados com tolerância angular de 25으, espaçamento de 90 metros (lag-distance) e até uma distância de 1500 metros (cutdistance).

A partir dos semivariogramas experimentais calculados, optou-se por utilizar o variograma experimental omnidirecional, ajustado ao modelo teórico Mattern com parâmetro de forma igual a 5 (kappa $=5)$ com raio de 90 metros (Figura 5).

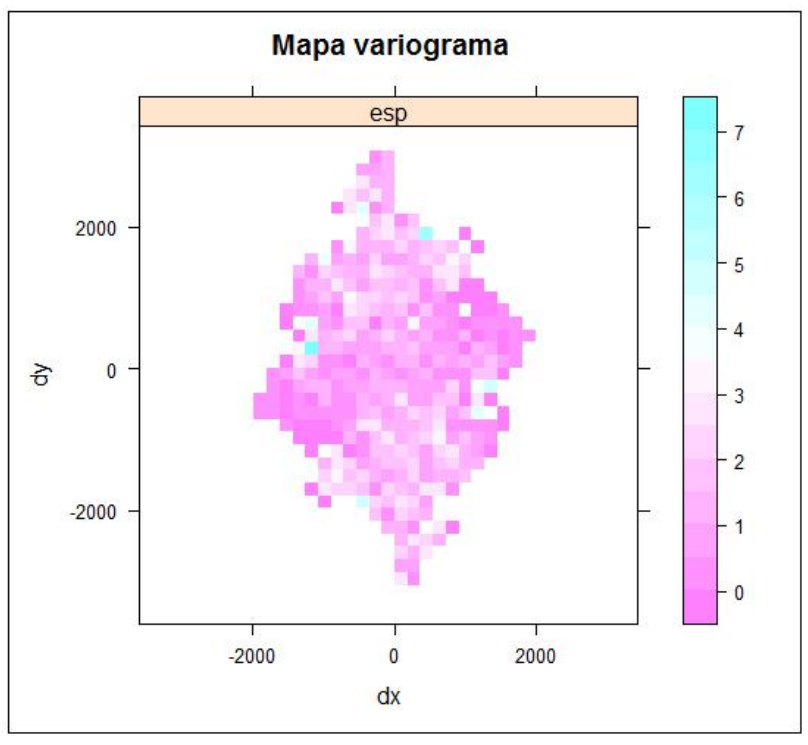

Figura 4 - Mapa variograma apresentando a semivariância dos dados em todas as direções.

\section{Predição geoestatística}

Para a predição, o método geoestatístico escolhido foi a krigagem por ser um procedimento de interpolação exato, ou seja, que leva em consideração todos os valores observados, não tendencioso pois atribui pesos a cada amostra de forma a minimizar a estimativa da variância (LANDIM, 2006), ser capaz fornecer a estimativa em um determinado ponto e a incerteza relacionada (OLIVER \& WEBSTER, 2014) e por permitir analisar variáveis regionalizadas.

De acordo com Landim (2010), o método de interpolação supracitado, quando o variograma é apropriado, é capaz de evitar ponderação arbitrária dos pontos amostrados e permite o estabelecimento de limites de confiança, ou seja, indica se os resultados são aceitáveis.

A Tabela 1 apresenta os parâmetros utilizados na construção do grid utilizados nas modelagens ressaltando que a modelagem está restrita aos limites da área urbanizada e por isso apresenta uma forma irregular. 

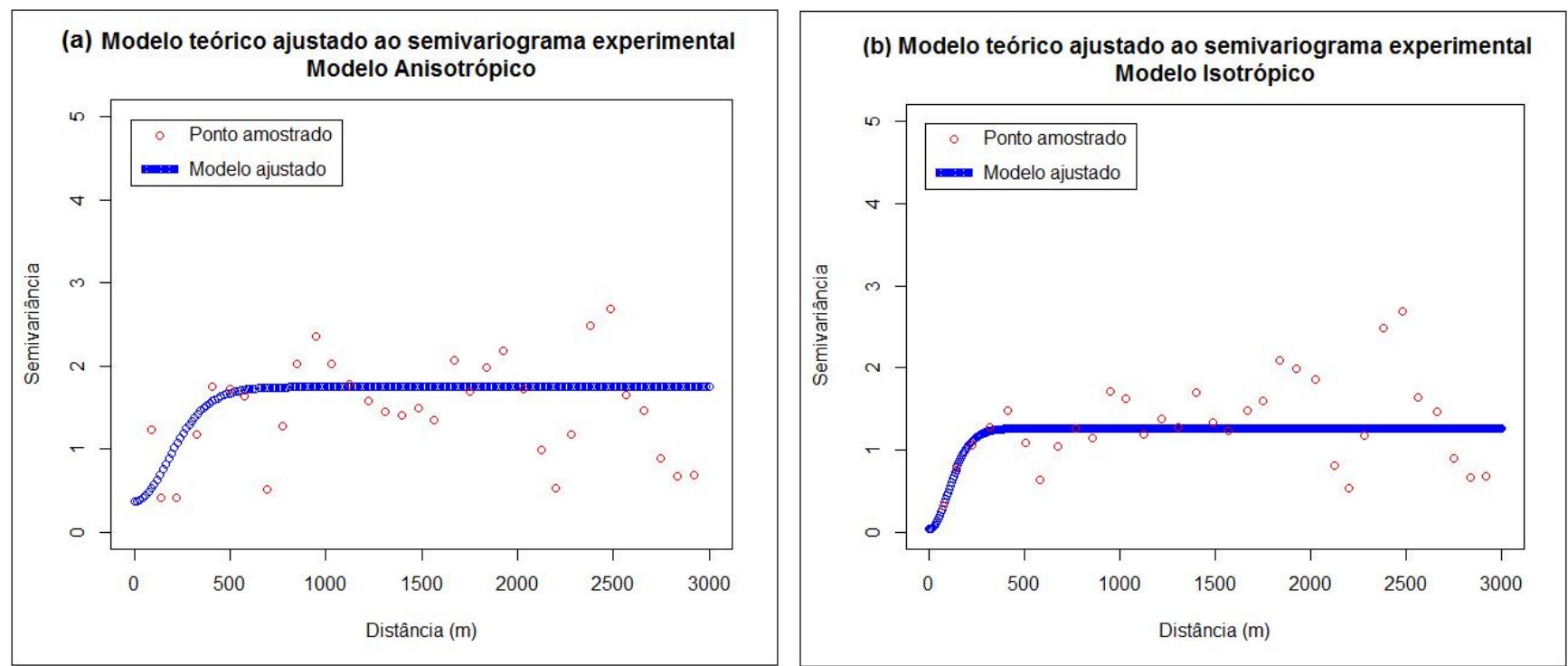

Figura 5 - Semivariograma experimental omnidirecional ajustado ao modelo teórico Mattern com parâmetro de forma igual a 5 (kappa=5).

Tabela 1 - Parâmetros utilizados no grid para a predição.. E: Espaçamento do grid; N: Número de pontos

\begin{tabular}{ccccc}
\hline Eixo & $\begin{array}{c}\text { Coordenada } \\
\text { mínima (UTM) }\end{array}$ & $\begin{array}{c}\text { Coordenada } \\
\text { máxima } \\
\text { (UTM) }\end{array}$ & $\mathrm{E}$ & $\mathrm{N}$ \\
\hline $\mathrm{X}$ & 825000 & 827200 & 10 & 220 \\
\hline $\mathrm{Y}$ & 8984500 & 8988000 & 10 & 350 \\
\hline
\end{tabular}

\section{Resultados e Discussão}

De posse das espessuras estimadas pelo processo de krigagem ordinária, utilizando o modelo isotrópico, realizou-se uma análise estatística univariada dos valores calculados para analisar o comportamento da variável estimada (Figura 6).

No modelo, o histograma e o valor de curtose demonstram que a distribuição é mais afunilada e concentrada e que os valores estimados estão bem semelhantes aos valores usados na estimativa, tanto em relação a amplitude nos dados quanto na forma da curva da distribuição acumulada. O boxplot identificou uma grande quantidade de valores como outliers devido ao fato da modelagem ter sido influenciada pelas poucas amostras de valores superiores à média.

Como resultado da modelagem realizada foi possível elaborar um mapa que apresenta a distribuição espacial das espessuras calculadas (Figura 7).

O modelo apresentou, na maior parte da área, valores de espessura estimada próxima da média e os valores de maior espessura ficaram restritos aos locais mais próximos as amostras que apresentaram tais valores. A transição entre as classes de espessura se apresentou de forma abruta.

Figura 7: Modelos isotrópico da espessura do solo na área de estudo

A pequena diferença entre os valores estimados pelos modelos pode ser devido a assimetria positiva devido a ocorrência de valores acima da média ou por não ter sido identificada Rtropia a partir da análise dos poucos dados disponíveis. Entretanto, o modelo apresentou bons resultados, pois estimou valores estimados mais próximos aos medidos e apresentou espessuras superiores somente nas áreas próximas aos pontos amostrados. 
Oliveira \& Antônio/Boletim Paranaense de Geociências 73-1 (2017) 46 - 53

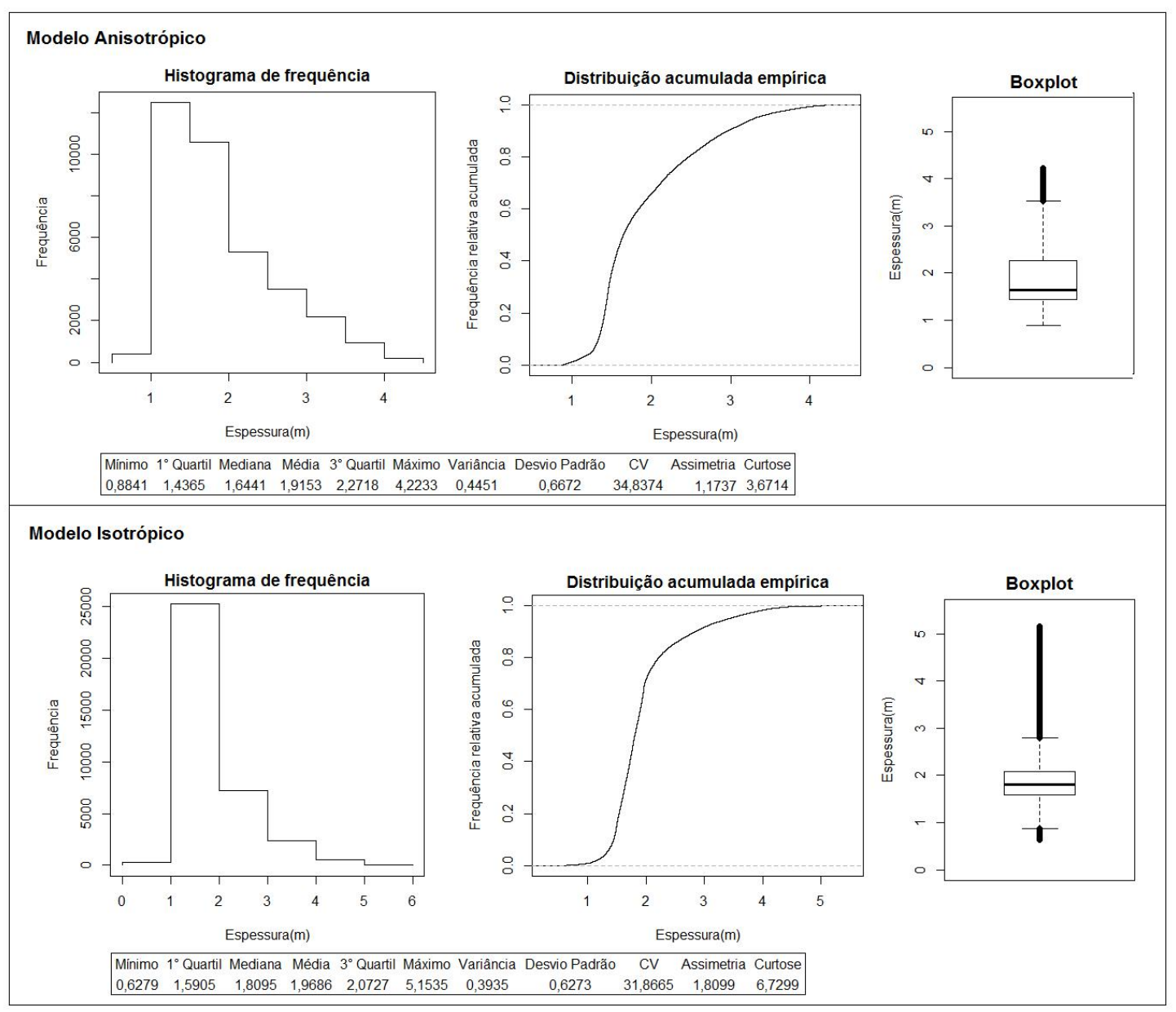

Figura 6 - Estatísticas univariadas dos valores preditos.

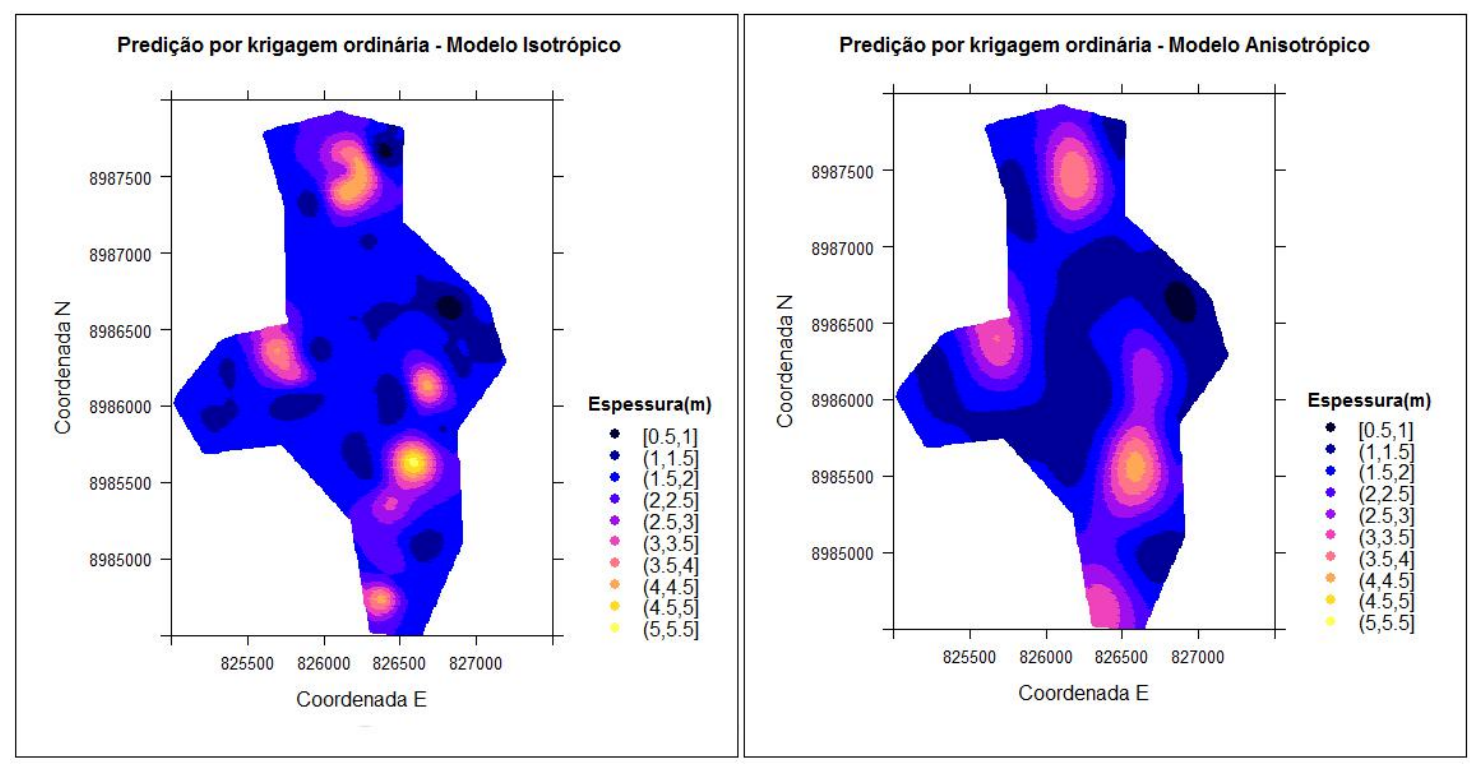

Figura 7 - Modelos isotrópico da espessura do solo na área de estudo. 


\section{Conclusão}

Com base nos resultados, é possível afirmar que a geoestatística se apresenta eficaz na produção de informações capazes de otimizar o planejamento e/ou complementar estudos geológico-geotécnicos e que a ferramenta de geoestatística, disponível no software utilizado, permitiu uma análise de qualidade das informações sem a necessidade da utilização de outros programas referentes a temática abordada.

É importante ressaltar alguns pontos quanto aos dados utilizados para estimar a espessura de solo na área de estudo.

$\mathrm{O}$ fato das investigações terem sido finalizadas em um metro e meio, em alguns locais por especificação do projeto, acarretou na concentração elevada desses valores no histograma o que, além de dificultar a modelagem geoestatística, não representa a espessura real do solo.

Todavia, a modelagem geoestatística é capaz de estimar dentro dos limites amostrados e com boa precisão a camada investigada, que é de extrema importância para projetos de engenharia.

De posse dos mapas temáticos, é possível predizer a espessura da camada de solo ao longo de uma via urbana e a variância das estimativas, prevendo custos adicionais de escavação em projetos de saneamento ou de reparos de redes de utilidades. Os mapas também ressaltam áreas que apresentam comportamentos diferentes, no caso do presente estudo são representadas pelas áreas que apresentam uma camada de solo superficial pouco espesso, que podem ser evitadas ou alvos de maior detalhamento dependendo do projeto a ser implantado.

Tendo dito isso, mesmo com informações limitadas, poucas amostras e irregularmente distribuídas, foi possível gerar mapas temáticos capazes de orientar planos de investigações futuras ou auxiliares na elaboração de projetos.

\section{Agradecimentos}

A empresa COHIDRO Consultoria Estudos e Projetos Ltda. pela disponibilização dos dados para a elaboração deste trabalho.

\section{Referências bibliográficas}

BRAGA L.P.V. 2014. Introdução a geoestatística com programas em R. Rio de Janeiro: E-papers, 132p.

LANDIM, P.M.B. 2006. Sobre Geoestatística e mapas. Terra e Didática, 2(1): 19-33. Disponível em: <http://ocs.ige.unicamp.br/ojs/terraedidatica/article/v iewFile/1008/442>. Acesso agosto de 2015.

LANDIM, P.M.B. 2010. Introdução aos métodos de estimação espacial para confecção de mapas Laboratório de Geomatemática, DGA/IGCE/UNESP, Rio Claro - Texto Didático 02. Disponível em: http://www.rc.unesp.br/igce/aplicada/DIDATICOS/LAN DIM/interpo.pdf>. Acesso em 17 de junho 2015.

OLIVEIRA, B.R.; BRANQUINHO, G.A. 2015. Mapeamento cartográfico do município de União dos Palmares - AL. Revista Eletrônica em Gestão, Educação e Tecnologia Ambiental. Santa Maria, 19 (3): 862-878.

OLIVER, M.A. \& WEBSTER R. 2014. A tutorial guide to geostatistics: Computing and modelling variogramas and kriging. Catena, 113: 56-69.

YAMAMOTO, J.K. 2001. Avaliação e classificação de Reservas Minerais. São Paulo: Editora da Universidade de São Paulo, 226p.

YAMAMOTO, J.K. \& LANDIM P.M.B. 2013. Geoestatística: conceitos e aplicações. São Paulo: Oficina de Textos, $215 p$.

Manuscrito ID 43417

Submetido em outubro de 2015

Aceito em julho de 2017 